\title{
Towards a model-based diagnostic instrument in electricity and magnetism - an example
}

\author{
Rasil Warnakulasooriya and Lei Bao \\ Dept. of Physics, The Ohio State University, 174 W.18 ${ }^{\text {th }}$ Avenue, Columbus OH 43210.
}

The identification of the contextual elements of a question is important. Based on our studies on the possible student mental models on certain concepts in electricity and magnetism, a set of multiple-choice questions were developed and tested. Examples of test questions and students' responses on one concept topic are discussed in detail. It is implied that measurement instruments developed based on a good understanding of the interactive relations between context features and the possible mental models can help identify important aspects of students' knowledge that are not available with current assessment tools.

\section{Introduction}

The context of a physics problem can significantly affect how a student responds to the problem. The understanding of the issue of context dependence serves an important role in curriculum development, test development and instruction. Our main aim has been to study contextual factors and apply the research in the development of a diagnostic test instrument in electricity and magnetism (E\&M).

We approach the study of contextual factors involved in E\&M by taking the concept of mental models (see below) as a basic premise. The framework of mental models provides a representational structure to the students' reasoning, which helps us analyze how students respond to particular questions posed in different contexts in different ways. Identifying mental models is particularly useful since model analysis $[1,2]$ can be employed for the analysis of data to help reveal students' knowledge state for a given concept and how these states shift depending on the context of the questions. Finally, our goal is to develop a multiple-choice model-based diagnostic instrument in electricity and magnetism.

The first step to develop a model-based diagnostic instrument is to identify the mental models that students have developed through their experience with the external world or through their formal learning. This is achieved by carrying out extensive qualitative research as described in our previous paper [3]. In [3] we reported the reasoning patterns on certain concepts that can be further explored to extract possible mental models. The present paper attempts to convey the findings of a detailed study on one concept topic, its possible models, and the contextual issues involved with it. However, we would first like to discuss what the phrase "mental model" means.

To quote Johnson-Laird [4], "The modern formulation of mental models is due to Craik [5]. He has argued that human beings translate external events into internal models and reason by manipulating [the resulting] symbolic representations. They can translate the resulting symbols back into actions or recognize a correspondence between them and external events".

Mental models help represent the features of the real world through perception or imagination. In physics, such imaginative models are often expressed through abstract mathematical formulations. Johnson-Laird has argued that although physical phenomena are expressed through verbal statements or mathematical formulations, comprehension should involve the construction of mental models for the processes that they represent. The predictive value of physics about the physical world stems through the construction of mental models [6]. According to Johnson-Laird [7], our ability to give explanations is intrinsically tied to understanding. In order to understand any phenomena we must have a working or an operational model of it. 
The term mental model has been used by researchers to mean different things under different contexts. One use of the term mental model is to mean a representation of some aspect of the external world (e.g., a vector field represented by a set of arrows). The other form is much more subtle since the model can be unstable, incomplete and could evolve. An example is provided by the common belief that heavier objects exert a larger force. Here, the common experience has been generalized although incorrect according to Newton's third law. However, such models could change due to formal instruction and under certain contextual features. The difference between the two usages could also be thought of as a difference between the levels of abstraction. In our study, we search for both forms of models and how and why such models come into existence.

We now turn to our main discussion. The questions given below were not selected randomly but were based partly on interviews and partly on certain hypotheses which we were eager to test. For example, Q1 (below) was given with the hypothesis that the charge at the tip of the arrows would trigger a 'direct pushing' toward the right although the charge is negative. This is a possibility if students are to carry certain representations from the classical mechanics domain to the domain of charges and electric fields [8]. Although, such a shift across domains could not be explicitly verified, the context of the questions gave rise to a rich set of reasoning including the charge been pushed to the right for more elaborate reasons. This helps us further identify certain dominant mental models. Similarly, Q3 was given with the hypothesis that it would trigger a 'no influence' response because of the discrete representation of the field lines whereas Q4 would trigger the opposite view. Again, our hypothesis was proved in part together with other forms of reasoning. Thus, we believe that the data can be used in addressing certain subtle features of a physical concept and its abstract representations in a manner that are at times overlooked.

\section{Concept topic - charged particle in an electric field}

The following questions (Q1-Q4) were given as a single survey to the students of a calculus-based electricity and magnetism class at The Ohio State University. The questions were given after the students have attended the lectures dealing with the necessary concepts. The survey was web-based and required them to give an explanation for each of their choices. Seventy one (71) students participated. In Q1, Q3 and Q4 the following question was posed followed by the respective diagrams. For these three, the choices given were the same.

Question (for Q1, Q3, Q4):

A negative charge is placed at rest in an electric field region (depicted by arrows) as shown below. Which way will the charge move? (In the actual survey the charge was shown in red.)

Q1.

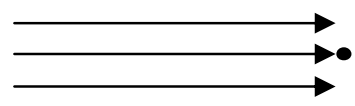

Q3.

Q4.

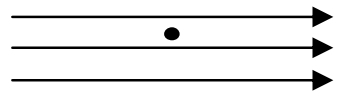

Choices (for Q1, Q3, Q4):

A It will not move at all.

B. It will move up.

C. It will move down.

D. It will move to the right.

E. It will move to the left.

In Q2 no figure was given. The question was posed as follows: A negative charge is placed at rest in an electric field region. The electric field points from left to right. Which way will the charge move?

For Q2 the same options A-E were given followed by an additional choice F: cannot say for sure.

We used a negative charge instead of a positive charge in order not to 'trivialize' the problem. We 
wanted to distinguish those who would answer consistently taking the negative charge into account and those who would answer it consistently taking the charge to be positive, which is the more psychologically 'friendly' charge. We believe that by giving a positive charge in the questions such identifications would not be possible. In this way we would have a better way of identifying those who are shifting based on the context of the different questions. About $50 \%$ of the students were consistent in answering all the questions correctly. Another $10 \%$ were consistent treating the given charge as positive. The rest fluctuated depending on the contextual elements. Simply put, the diagrammatic representations were interpreted as they meet the eye rather than as abstract and idealized representations.

About $18 \%$ selected choice A to Q1. The charge placed at the end of the arrows in Q1 prompted the response of the 'end of the electric field region' ("it will not move since it is not 'in' the electric field"). The 'end of the field' also gave rise to the choice D. One argument goes as follows: "The electric field points from positive charge to the negative charge [the positive and the negative charge the student refers to is the way the student 'visualizes' a field line and is not the negative test charge in question]. The arrows stop just before the negative charge [here, the negative charge is the test charge in question] so I am assuming the field region borders the apex of the arrows and indicates the location of the negative charge causing the electric field. Like charges repel. The left side is theoretically closest to the negative charge. [The test charge] Moving right is due to the negative charges toward the left". Here the student has taken the phrase "electric field lines go from positive to the negative charges' to imagine a set of negative charges at the tip of the arrows which would in turn repel the negative test charge.

In Q2 the absence of a picture gave way to the answer F which $10 \%$ of the students selected ("it depends on the position of the charge"). We also observe a 10\% increase (not necessarily the same students as in the previous case) in choice A for Q3 where the charge is considered not within the influence of the electric field lines ("no field lines appear to be contacting the charge"). However, this particular representation has also given rise to arguments like "[the charge] is actually in the field...will be attracted to the positive side, which is to the left". The distribution of answers to Q1 and Q4 did not differ much. It is surprising that there is only 3\% drop from Q1 to Q4 with regard to choice A. On careful examination of student reasoning it seems that 'symmetry' played a part in Q4. Due to the position of charge in Q4 the leftright and up-down regions look symmetrical in the picture with the charge at the center. This leads to a field surrounding the charge homogeneously and isotropically thus 'balancing out the influence of the field on the charge' ("it [the charge] will not move because a constant field is around it and therefore canceling out any tendencies to move"). The asymmetric arguments gave rise to an increase of choice $\mathrm{C}$ by $\sim 6 \%$ in Q3 as compared to the others. Since there are two field lines below the charge as opposed to one line above it, the charge would be more influenced by the lower region 'dragging' the charge down ("It [the charge] will move down. The field is stronger beneath the charge"). In both cases the direction of the field lines and its meaning as treated in the previous questions were abandoned and given a new interpretation.

\section{Discussion}

Half of the students were consistent in their choices where as $\sim 40 \%$ varied based on the context elements. In the questions the predominant context element could be identified as the position of the negative charge with respect to the depicted electric field lines. Under certain contexts it may be argued that the physicist's conception, depiction and the interpretation of the field lines as an operational or a working model translated to a highly physical model in the students' mind. This could be easily seen in the context of Q3. There, it becomes apparent that some students do not visualize a continuum of lines in between the ones that are shown. In Q1 the arrows were not taken as depicting a constant electric field region which extends far beyond the boundaries of the picture but rather where positive and negative source charges are placed which produces that field. 
Students who argued in this manner reached the conclusion that the arrow tips represent negative charges therefore repelling the negative test charge to the right. They failed to realize that having charges at the tips and simultaneously considering a void to the right of these charges are inconsistent. The same visualization of source charges was not put to use in the context of Q4. There, 'symmetry' took precedence over other models in students who were susceptible to the context elements. In this situation the field lines become synonymous with force lines and a 'balancing of forces' on the charge from the lines that surround it 'symmetrically' is triggered without regard to what the arrows stand for.

It is possible that presenting the questions individually could result in a different distribution. But then it does not reveal the advantages of presenting a collection of questions designed to probe students' understanding more thoroughly. We believe that having Q2 with its additional choice, F, adds a whole new dimension to how students view the questions in relation to each other. It is also important to note that not having a diagram in Q2 did not change the reasoning of students who answered consistently. But what would be the reasoning patterns if we present Q2 alone? This we will pursue in future studies. For a diagnostic instrument, incorporating such a set of questions may help instructors to derive a better understanding about students' knowledge states and also address the important issue of the very nature of science as projections of the physical world to abstract planes. This may help the students leave the classroom with physical and working models which are consistent with each other and which would lead to the 'correct physics' when the concepts are in need of application.

\section{Acknowledgements}

We thank Dr. Frank C. De Lucia for his support in conducting the surveys and the PER group at the OSU for helpful suggestions. This work is supported in part by NSF grant \#REC0087788 .
[1] L. Bao and E.F. Redish, "Model Analysis: Assessing the dynamics of student learning". Submitted to Cognition and Instruction. http://www.physics.ohio-state.edu/ lbao/

[2] L. Bao, K. Hogg and D. Zollman, "Model analysis of fine structures of student models: An example with Newton's third law". Am. J. Phys. 70 (7), 2002, pp 766-778.

[3] R. Warnakulasooriya and L. Bao, "Preliminary studies on students' understanding of electricity and magnetism for the development of a modelbased diagnostic instrument", Proceedings of the 2001 Physics Education Research Conference, pp 127-130.

[4] P.N. Johnson-Laird, Mental Models, In M. I. Posner (Ed.), Foundations of cognitive science, Cambridge, MA: MIT Press (1989), pp 469-499.

[5] K.Craik The Nature of Explanation, Cambridge: Cambridge University Press (1943).

[6] I.M. Greca and M.A. Moreira, Mental models, conceptual models, and modeling, Int. J. Sci. Educ, 22 (1), 2000, pp 1-11.

[7] P.N. Johnson-Laird, Mental Models: Towards a Cognitive Science of Language, Inference, and Consciousness, Cambridge: Cambridge University Press (1983).

[8] I. Galili, Mechanics background influences students' conceptions in electromagnetism, Int. J. Sci. Educ, 17 (3), 1995, pp. 371-387. 